\title{
Las transiciones en el uso de alcohol en una cohorte de nacimiento-sexo en la población general: el proyecto transversal PEGASUS-Murcia
}

\author{
Birth-sex cohort alcohol use transitions in the general \\ population: the cross-sectional PEGASUS-Murcia project
}

\author{
Mathilde M. Husky*, Chrianna Bharat**, Gemma Vilagut***, Diego Salmerón****, \\ Salvador Martínez*****, Carmen Navarro******, Jordi Alonso***, Ronald C. Kessler*******, \\ FERnando NAVARro-MateU $* * * * * * * *$.
}

\begin{abstract}
* Institut Universitaire de France, Université de Bordeaux, Laboratoire de Psychologie EA4139, Burdeos. Francia; ** National Drug and Alcohol Research Centre, UNSW Sydney, Sydney, Australia; *** IMIM-Institut Hospital del Mar d'Investigacions Médiques, Barcelona, España. CIBER in Epidemiology \& Public Health (CIBERESP), España; **** Departamento de Ciencias Sociosanitarias, Universidad de Murcia, CIBER in Epidemiology \& Public Health (CIBERESP), IMIB-Arrixaca, Murcia, España; ***** Instituto de Neurociencias UMH-CSIC, Alicante, España; ****** Servicio de Epidemiología, Consejería de Sanidad y Política Social, Murcia, CIBER in Epidemiology \& Public Health (CIBERESP), IMIB-Arrixaca, España; ******* Department of Health Care Policy, Harvard Medical School, Boston, Estados Unidos. ******** Unidad de Docencia, Investigación y Formación en Salud Mental (UDIF-SM), Servicio Murciano de Salud. Departamento de Psicología Básica y Metodología. Universidad de Murcia, CIBER in Epidemiology \& Public Health (CIBERESP), IMIB-Arrixaca, Murcia, España.
\end{abstract}

\section{Resumen}

Examinar el impacto potencial de la prevalencia de uso de alcohol en una cohorte de nacimiento-sexo en el inicio y progresión del uso de alcohol en el proyecto PEGASUS-Murcia, encuesta transversal en una muestra representativa de adultos no institucionalizados de Murcia (España). Se entrevistaron personalmente a 1.459 adultos sobre consumo de alcohol a lo largo de la vida, trastornos por uso de alcohol (criterios DSM-IV) y remisión utilizando la Entrevista Diagnóstica Internacional Compuesta (CIDI 3.0). Se calcularon estimaciones de tablas de vida basadas en las funciones de supervivencia para la edad de inicio en el uso de alcohol y su remisión en modelos de supervivencia de tiempo discreto. Casi nueve de cada diez adultos tuvieron una historia de uso de alcohol a lo largo de la vida. Entre ellos, $84,3 \%$ desarrolló un uso regular (> 12 bebidas por año) y 5,5\% y $1,6 \%$ cumplieron criterios DSM-IV de Abuso y Dependencia de alcohol, respectivamente. A los 18 años, 70,9\% había usado alcohol, $50,2 \%$ de forma regular, con un aumento brusco en adultos jóvenes (90,8\% a los 22 años). El uso de alcohol de la cohorte de nacimientosexo se asoció significativamente con mayores probabilidades para todas las transiciones examinadas, excepto en la transición uso-abuso. Se detectan períodos sensibles con transiciones rápidas a niveles más altos de uso de alcohol. Las experiencias de cohortes en todas las etapas del consumo de alcohol son importantes. Estos resultados podrían contribuir a la predicción de las tendencias poblacionales de los problemas con el alcohol en España.

Palabras clave: Alcohol; Abuso; Cohorte; Dependencia; Remisión.

\begin{abstract}
To examine the potential impact of prevalence of alcohol use in a birth-sex cohort on subsequent initiation and progression of alcohol use in the PEGASUS-Murcia project, a cross-sectional survey of a representative sample of non-institutionalized adults in Murcia (Spain). Data on lifetime history of alcohol use, DSM-IV use disorders, and remission were collected from 1,459 adults using faceto-face interviewers based on the Composite International Diagnostic Interview (CIDI 3.0). Life-table estimates based on survival functions for alcohol use age-of-onset and remission were used as time-varying predictors of subsequent individual-level alcohol use in discrete-time survival models. Nearly nine out of ten adults had a lifetime alcohol use history at time of interview. Of these lifetime users, $84.3 \%$ became regular users (>12 drinks a year) and 5.5-1.6\% went on to meet criteria for DSM-IV alcohol abuse or dependence, respectively. By the age of $18,70.9 \%$ of respondents had used alcohol, and one half $(50.2 \%)$ had used regularly. Regular use sharply increased during early adulthood to reach $90.8 \%$ by age 22 . Birth-sex cohort alcohol use was significantly and positively associated with increased odds of all subsequent transitions examined except for the transition from use to abuse. The findings highlight sensitive periods with rapid transitions to higher levels of alcohol use and emphasize the importance of cohort experiences in the full spectrum of stages of alcohol use. These results may contribute to predicting population-levels trends in alcohol-related problems in Spain.

Keywords: Alcohol; Abuse; Cohort-use; Dependence; Remission.
\end{abstract}

Recibido: Noviembre 2017; Aceptado: Julio 2018.

Enviar correspondencia a:

Fernando Navarro-Mateu, MD, PhD. Unidad de Docencia, Investigación y Formación en Salud Mental (UDIF-SM). Servicio Murciano de Salud. c/ Lorca, n 58. 30120-El Palmar (Murcia). Spain. Email: fernando.navarro@carm.es 
S egún el Observatorio Español de la Droga y las Toxicomanías (2017), entre 2009-2015, más del 90\% de los españoles habían utilizado alcohol al menos una vez en su vida y el 9,3\% de la población consumía alcohol a diario (Gual et al., 2016). En España, el consumo de alcohol per cápita entre los individuos de 15 o más años de edad se estima en 11,2 litros por año (Organización Mundial de la Salud- OMS, 2014) y se considera que el alcoholismo es un importante problema de salud pública (Pérez, 2002). Desde mediados de 1970, el consumo general de alcohol disminuyó debido principalmente a una reducción en el consumo de vino. A partir de 2010, el 50\% del consumo registrado de alcohol era cerveza, seguida de bebidas destiladas $(28 \%)$ y vino $(20 \%)$. Como sucede en España, la mayor parte de los adultos que viven en culturas occidentales han consumido alcohol en algún momento de su vida (Degenhardt et al., 2008), pero solamente una pequeña porción desarrolla un Trastorno por Consumo de Alcohol (TCA) (Demyttenaere et al., 2004). Teniendo en cuenta la gravedad de la carga de la enfermedad relacionada con el alcohol (Rehm, Gmel y Gual, 2013; Rehm et al., 2009), se han realizado numerosas investigaciones para identificar los factores de riesgo de transición de uso temprano a estadios subsecuentes de uso de alcohol.

Ser varón, un nivel educativo más bajo, el origen étnico, la exposición a experiencias traumáticas, trastornos mentales previos, y la edad de inicio en el uso de alcohol se han asociado a mayor riesgo de transición de uso de alcohol a TCA (DeWit, Adlaf, Offord y Ogborne, 2000; Flórez-Salamanca et al., 2013; Grant, 1997; Kalaydjian et al., 2009; Lee et al., 2009; Lev-Ran, Imtiaz, Rehm y Le Foll, 2013; Lopez-Quintero et al., 2011; Oberleitner, Smith, Weinberger, Mazure y McKee, 2015; Probst, Moyo, Purshouse y Rehm, 2015; Silveira et al., 2011; Suliman, Seedat, Williams y Stein, 2010; Werner et al., 2016). Además, se ha demostrado que factores contextuales, incluyendo el uso de sustancias entre iguales, el período, la cohorte de nacimiento-sexo y la edad afectan tanto el uso de alcohol de una determinada población (Degenhardt, Stockings, Patton, Hall y Lynskey, 2016; Grant, 1997; Rehm et al., 2015) como las transiciones de uso hacia trastornos (Grant, 1997). Sin embargo, el papel del uso de la cohorte de nacimiento-sexo en todo el espectro de transiciones de uso a TCA y de TCA a la remisión aún no está claro. Los estudios que han examinado los factores asociados a la trayectoria completa de uso de alcohol han proporcionado evidencias de riesgo diferencial de los factores contextuales según la etapa de progresión (Abdin, Subramaniam, Vaingankar y Chong, 2013; Carballo, Fernandez-Hermida, Secades-Villa y García-Rodríguez, 2008; Carballo et al., 2008; Kalaydjian et al., 2009; Lee et al., 2009; Silveira et al., 2011), reforzando la necesidad de incluir todo el espectro de transiciones de alcohol al examinar los factores de riesgo específicos.
El presente estudio examina el rol de uso de la cohorte en la historia natural de uso de alcohol y los trastornos de uso en una muestra representativa de la población general de Murcia, una de las 17 comunidades autónomas de España. Los objetivos específicos son identificar la edad de inicio y el tiempo para transitar entre varios estadios de uso de alcohol en la población general de Murcia y estimar la asociación del uso de alcohol de la cohorte nacimiento-sexo con la probabilidad de transición a uso regular de alcohol, TCA y a la remisión del abuso.

\section{Método}

\section{Procedimiento y participantes}

El proyecto PEGASUS-Murcia ("Psychiatric Enquiry to General Population in Southeast Spain-Murcia”) es una encuesta trasversal de la población general que forma parte de la Iniciativa de Encuesta de la Salud Mental Mundial (WMH) (http://www.hcp.med.harvard.edu/wmh/). El estudio se realizó entre 2010 y 2012 y fue diseñado para recopilar datos sobre la prevalencia, edad de inicio, carga, tratamiento, y correlatos de trastornos mentales comunes en una muestra representativa de la población general de adultos residente en la región de Murcia (Navarro-Mateu et al., 2013b; Navarro-Mateu et al., 2015). La tasa de respuesta general fue del 67,7\%. Después de obtener autorización para la participación, entrevistadores entrenados llevaron a cabo entrevistas en persona asistidas por ordenador a 2.621 adultos de 18 o más años. Como se describe en detalle previamente (Navarro-Mateu et al., 2013b), las entrevistas se dividen en dos partes para reducir la tarea del participante: Parte 1 y Parte 2. La parte 1 se administró a todos participantes e incluía la evaluación diagnóstica principal de trastornos del estado afectivo y de la ansiedad. La parte 2 contenía la evaluación de trastornos mentales adicionales, incluyendo los trastornos del uso de alcohol, y se administró a los encuestados que referían tener síntomas emocionales y de ansiedad, y a una submuestra aleatoria de los que no habían referido tales síntomas. El presente estudio se basa en la muestra $(n=1.459)$ de la Parte 2 de quienes se obtuvieron los datos sobre el uso de alcohol y TCA. El protocolo fue aprobado por la Comisión de Investigación de Ética Clínica del Hospital Universitario Virgen de la Arrixaca de Murcia. El artículo ha sido escrito siguiendo las recomendaciones de la guía STROBE (Strengthening the Reporting of Observational Studies in Epidemiology) (von Elm et al., 2007).

\section{Medidas: Uso de alcohol y estadios de uso}

La adaptación española (Navarro-Mateu et al., 2013a) de la versión CAPI (Computer Assisted Personal Interviewing o Entrevista personal asistida por ordenador) de la Entrevista Diagnóstica Internacional Compuesta de la Organización Mundial de la Salud 3.0 (WHO-CIDI, en 
adelante CIDI) (Kessler y Ustun, 2004) se utilizó para determinar la presencia de TCA según el Manual Diagnóstico y Estadístico de Trastornos Mentales, cuarta edición (DSM-IV) (American Psychiatric Association, 2000). El uso de alcohol se definió como haber consumido alguna vez una bebida alcohólica estándar, incluyendo cerveza, vino, refrescos de vino, o licor fuerte. El uso regular fue definido como haber consumido 12 o más bebidas alcohólica en un período de un año. Se hicieron preguntas sobre el abuso y la dependencia de alcohol a todas las personas que, en el año en que más bebieron, consumían alcohol por lo menos una vez por semana, o bebieron cinco o más copas por día en los días en que bebían. La remisión se definió como la ausencia de trastorno relacionado con los síntomas durante más de 12 meses antes de la entrevista. Además, se recogieron informes retrospectivos de la edad-de-inicio con respecto a cada etapa de uso de alcohol.

\section{Análisis de los datos}

Todos los análisis se basaron en datos ponderados, teniendo en cuenta el diseño muestral complejo, con estratificación y múltiples etapas de muestreo, como se describe en detalle en otra parte (Navarro-Mateu et al., 2013b). Brevemente, los pesos de muestreo se aplicaron para controlar las probabilidades diferenciales de selección en cada etapa de muestreo, y los pesos de post estratificación se aplicaron para garantizar que los datos fueran representativos de la población general regional según los datos del padrón disponibles de la región de Murcia en 2010. Los individuos de la Parte 2 recibieron un peso de muestreo adicional con el fin de controlar el muestreo diferencial de individuos con problemas de salud mental y asegurar la representatividad de la submuestra de Parte 2. La prevalencia de por vida se estimó como la proporción de todos los encuestados que habían cumplido los criterios para un determinado trastorno en sus vida hasta la edad que tenían en la entrevista. Las estimaciones de tablas de vida (actuariales) de las funciones de supervivencia para la edad de inicio y remisión se calcularon con el procedimiento PROC LIFETEST en SAS Versión 9.4.

La asociación entre las transiciones por los estadíos de alcohol y la prevalencia vida de uso de alcohol del nivel de la cohorte nacimiento-sexo, controlando por los datos socio-demográficos básicos, se evaluaron con las odds-ratios y las estimaciones de los intervalos de confianza de modelos de supervivencia múltiple en tiempos discretos con el procedimiento PROC SURVEYLOGISTIC en SAS, con persona-año como la unidad de análisis y una función de enlace logístico. Las personas-año se definieron desde los seis años de edad para el modelo de inicio de uso, desde la edad de comienzo de abuso para la remisión de abuso, y todos los demás modelos desde la edad de comienzo del primer estadio hasta la edad de comienzo del segundo (dependiendo del modelo) o la edad en la entrevista (para los casos censurados). No hubo un número suficiente de casos de dependencia para analizar las transiciones hacia o desde la dependencia del alcohol.

Se definió una variable contextual para representar el nivel de uso de alcohol en la cohorte de nacimiento-sexo de un individuo para estimar el efecto de los cambios en el uso de alcohol a lo largo del tiempo. La cohorte de nacimiento se definió como el año de nacimiento +/- 5 años, creando una cohorte específica por sexo con una amplitud de 11 años alrededor de cada año de nacimiento. La extensión de las cohortes se redujeron para los menores de 23 años para garantizar la simetría del año de nacimiento lo más posible, y se censuraron las cohortes de 65 años en adelante. La covariable modelada fue una estimación cambiante en el tiempo de la proporción de personas $(/ 10)$ en la cohorte de nacimiento-sexo del individuo que ha usado alcohol en el año previo, para cada persona- año.

Otras variables consideradas en todos los modelos de transición por uso/trastorno de uso fueron el sexo, edad de inicio de uso (excepto en los modelos de inicio de uso), definida como los terciles temprano, medio o tardío, el nivel de educación como variable cambiante en el tiempo, y el grupo de edad de la unidad persona-año $(<=14,15-17$, 18-20, 21-24, 25-29 and 30+). Además de éstos, los modelos de la remisión de abuso también incluían la velocidad de la transición de uso a abuso y los años que llevaba con el trastorno, así como agrupaciones alternativas de edad de persona-año (<=18, 19-20, 21-22, 23-24, 25-29, 30-39 and 40+). Se realizaron pruebas de significación con la prueba de $\chi^{2} \mathrm{de}$ Wald utilizando las matrices de varianza-covarianza de los coeficientes obtenidas con linearización en series de Taylor para controlar el diseño muestral complejo. Todas las pruebas de significación se evaluaron al nivel de 0,05 bilateral.

\section{Resultados}

\section{Prevalencia de uso de alcohol, trastornos de uso de alcohol y remisión}

La gran mayoría de los encuestados $(89,4 \%)$ consumió alcohol al menos una vez en su vida, con un $75,4 \%$ de encuestados que usaba alcohol regularmente ( $\geq 12$ copas un año) (Tabla 1). La prevalencia vida de abuso de alcohol según el DSM-IV (sin dependencia) fue de 4,9\% y de dependencia del alcohol fue de 1,5\%. Entre los que usaban alcohol, la gran mayoría de los adultos lo hacía de forma regular $(84,3 \%)$, y sólo una pequeña porción desarrolló abuso $(5,5 \%)$ o dependencia de alcohol $(1,6 \%)$. La mayoría de los encuestados con trastorno vida de abuso $(85,3 \%)$ o dependencia $(79,4 \%)$ había remitido en el momento de la entrevista.

\section{Edad de inicio para los estadios de uso de alcohol}

Las curvas de edad de inicio acumulada (age of onset-AOO) para todos estadios de uso de alcohol se presentan en la Figura 1. Al cumplir la edad de 18 años, el 70,9\% 
Mathilde M. Husky, Chrianna Bharat, Gemma Vilagut, Diego Salmerón,

Salvador Martínez, Carmen Navarro, Jordi Alonso, Ronald C. Kessler, Fernando Navarro-Mateu

Tabla 1. Prevalencia de uso de alcohol, trastornos de uso de alcohol según el DSM-IV y remisión en Murcia.

\begin{tabular}{lccc}
\hline & $\mathrm{n}$ & $\%^{\mathrm{a}}$ & $\mathrm{ES}$ \\
\hline Prevalencia-vida & & & \\
\hline Uso & 1459 & 89,4 & 1,3 \\
Beber $\geq 12$ copas al año & 1459 & 75,4 & 2,4 \\
Abuso sin dependencia & 1459 & 4,9 & 0,7 \\
Dependencia & 1459 & 1,5 & 0,5 \\
Remisión de abuso & 1459 & 4,2 & 0,8 \\
Remisión de dependencia & 1459 & 1,2 & 0,5 \\
& & & \\
Prevalencia condicional & & & \\
\hline Beber $\geq 12$ copas al año | uso & 1272 & 84,3 & 1,9 \\
Abuso sin dependencia | uso & 1272 & 5,5 & 0,7 \\
Dependencia | uso & 1272 & 1,6 & 0,6 \\
Remisión de abuso & & & \\
| abuso sin dependencia a lo largo de la vida & 73 & 85,3 & 7,6 \\
Remisión de dependencia & & & \\
| dependencia a lo largo de la vida & 20 & 79,4 & 13,5 \\
\hline
\end{tabular}

Nota. ES $=$ Error estándar. $\mathrm{n}=$ El número total no ponderado de los encuestados que respondieron a preguntas sobre el uso de alcohol de prevalencia de porvida, y el número total no ponderado de los encuestados en la cohorte condicional para la prevalencia condicional. a Las estimaciones de prevalencia se basan en los datos ponderados.



Figura 1. Curvas de edad de inicio de estadios de alcohol en el proyecto PEGASUS-Murciaa,b

Nota. ${ }^{\text {a }}$ Las estimaciones a lo largo de la vida de las funciones de supervivencia se basan en los datos ponderados de la Parte II (muestra total de los 1.459 encuestados) e incluyen los encuestados con y sin el diagnóstico específico, donde edad de inicio para este último caso es censurado en la edad de entrevista. Las estimaciones se escalan hasta llegar a $100 \%$; ' ${ }^{\mathrm{b}}$ Los encuestados con datos perdidos de edad de inicio de la remisión de abuso $(n=10)$ se excluyeron de la curva de remisión de abuso.

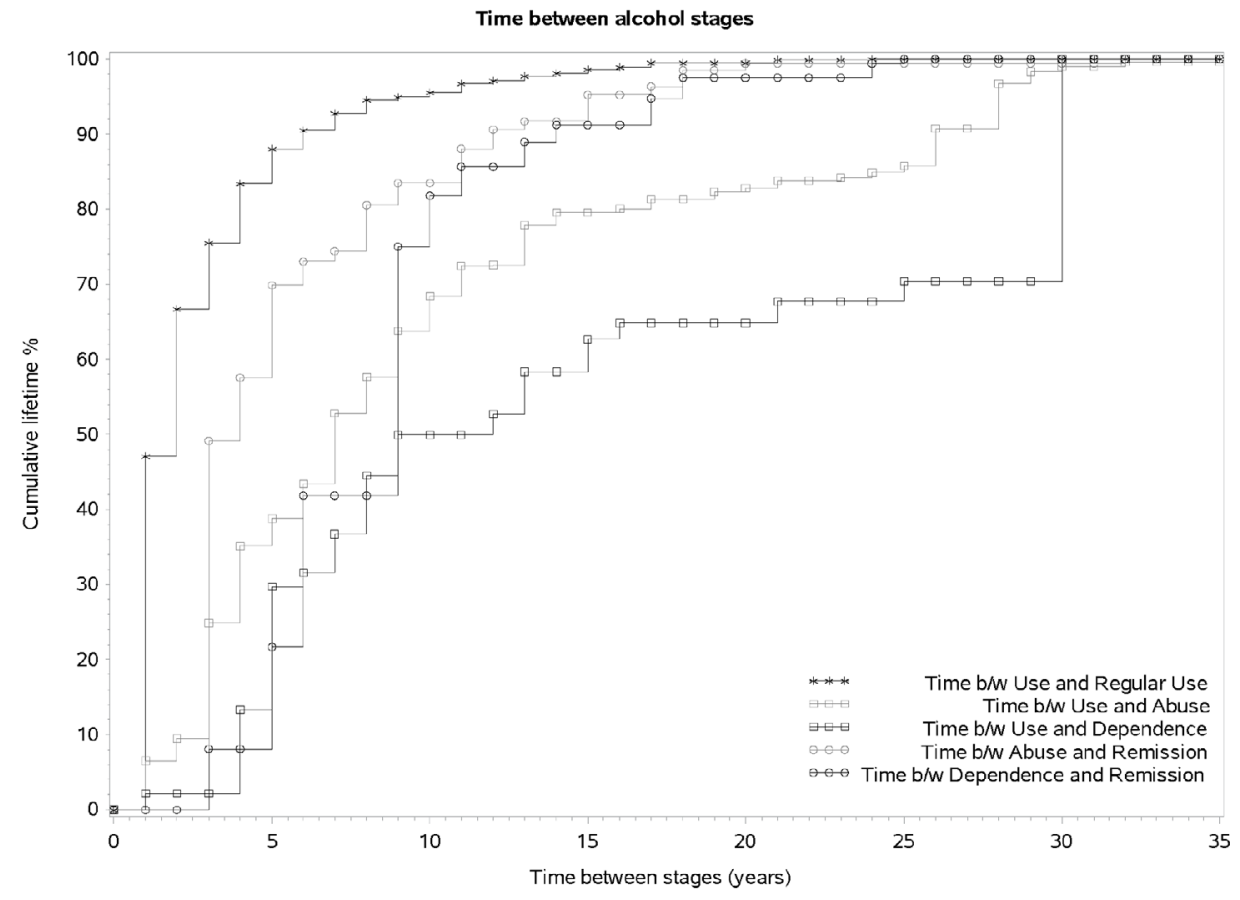

Figura 2. Tiempo entre estadios de alcohol en el proyecto PEGASUS-Murcia ${ }^{\text {un,b }}$


sólo incluye los encuestados con un diagnóstico en el segundo de los estadios. ${ }^{b}$ Los encuestados con datos perdidos de edad de inicio de la remisión de abuso $(\mathrm{n}=10)$ se excluyeron de la curva de remisión de abuso. 
de los consumidores de alcohol y el $50,2 \%$ de los que lo consumen de forma regular habían iniciado dichos estadios. El uso regular aumenta marcadamente durante el período adulto temprano, alcanzando el $90,8 \%$ al cumplir los 22 años. La AOO media para el abuso de alcohol (23 años) se alcanza dentro de los 5 años de la AOO mediana para el uso regular (18 años). Tres de cada cuatro casos de abuso de alcohol $(75,3 \%)$ se observan al cumplir los 31 años. Un tercio de los casos de dependencia del alcohol se observan antes de los 22 años, alcanzando el 55,4\% cuando cumplen 31 años y el 95,0\% cuando cumplen los 43 años. Las curvas AOO de remisión muestran que el $50 \%$ de las remisiones de abuso de alcohol se producen cerca de los 29 años mientras que el $50 \%$ de remisiones de dependencia del alcohol se producen alrededor de los 48 años y el $100 \%$ cuando se cumplen 51 años.

\section{Tiempo desde la transición a través de estadios de implicación con el uso de alcohol}

La transición más rápida se encuentra en la transición de uso inicial al uso regular (Figura 2). Al año de su primer contacto con el alcohol, el 47,1\% de los usuarios se había convertido en usuario regular y a los tres años, el 75,5\% se había convertido en usuario regular. La transición desde el primer uso a TCA es más lento; El 52,7\% de las transiciones de uso a abuso ocurren dentro de los 7 años, y el 52,7\% de las transiciones de uso a dependencia ocurren dentro de los 12 años. La transición desde TCA hasta el inicio de la remisión fue más rápida para abuso de alcohol que para dependencia del alcohol. Dentro de los tres años desde el inicio del trastorno, el 49,1\% de los casos de abuso de alcohol y sólo el 8,1\% de los casos de dependencia del alcohol había remitido. Dentro de los 9 años desde el inicio del trastorno, la mayoría de los casos de abuso $(83,5 \%)$ y de dependencia $(75,0 \%)$ había remitido.

\section{Transiciones a través de los estadios de uso de alcohol}

La Tabla 2 presenta el análisis de supervivencia múltiple de tiempo discreto que examina las covariables de la transición desde no-uso a uso, uso a uso regular, uso a abuso, y uso regular a abuso en un determinado año. Las transiciones desde uso a abuso y desde uso regular a abuso fueron significativamente más habituales en los varones. El uso de alcohol de la cohorte nacimiento-sexo se asociaba significativamente con un incremento de las probabilidades de todas las transiciones examinadas con la excepción de la

Tabla 2. Asociaciones ajustadas de variables socio-demográficas con transiciones entre estadios de uso de alcohol a lo largo de la vidal TCA en Murcia.

\begin{tabular}{|c|c|c|c|c|c|c|c|c|c|}
\hline \multirow[t]{2}{*}{ Variable } & \multirow[t]{2}{*}{ Categoría } & \multicolumn{2}{|c|}{ Inicio de uso } & \multicolumn{2}{|c|}{$\begin{array}{c}\text { Uso a beber } \\
\geq 12 \text { copas al año }\end{array}$} & \multicolumn{2}{|c|}{$\begin{array}{c}\text { Uso a abuso } \\
\text { (sin dependencia previa) }\end{array}$} & \multicolumn{2}{|c|}{$\begin{array}{c}\text { Uso regular a abuso } \\
\text { (sin dependencia previa) }\end{array}$} \\
\hline & & OR & IC $95 \%$ & OR & IC $95 \%$ & OR & IC $95 \%$ & OR & IC $95 \%$ \\
\hline Sexo (Ref: Mujer) & $X_{1}^{2}[p]$ & 1,92 & {$[0,165]$} & $<0,10$ & {$[0,998]$} & $8,62^{\star \star}$ & {$[0,003]$} & $5,63^{\star \star}$ & {$[0,018]$} \\
\hline \multirow{2}{*}{$\begin{array}{l}\text { Porcentaje de uso de alcohol } \\
\text { de la cohorte nacimiento-sexo }\end{array}$} & $\begin{array}{l}\text { Uso de la } \\
\text { cohorte }\end{array}$ & $1,50^{\star}$ & $(1,44-1,57)$ & $1,16^{*}$ & $(1,09-1,24)$ & 1,43 & $(0,98-2,10)$ & $1,46^{*}$ & $(1,06-2,01)$ \\
\hline & $X_{1}^{2}[p]$ & $333,94^{\star \star}$ & {$[<0,001]$} & $19,32^{\star \star}$ & {$[<0,001]$} & 3,38 & {$[0,066]$} & $5,50^{\star \star}$ & {$[0,019]$} \\
\hline \multirow{4}{*}{ Nivel educativo (Ref: Alto) } & Estudiante & 0,67 & $(0,14-3,26)$ & $0,41^{*}$ & $(0,19-0,87)$ & 2,80 & $(0,66-11,85)$ & 2,63 & $(0,63-11,02)$ \\
\hline & Bajo & 0,53 & $(0,12-2,34)$ & $0,38^{\star}$ & $(0,16-0,92)$ & 0,65 & $(0,25-1,66)$ & 0,67 & $(0,27-1,68)$ \\
\hline & Bajo-medio & 0,66 & $(0,11-3,92)$ & $0,37^{\star}$ & $(0,18-0,73)$ & 2,98 & $(0,68-13,04)$ & 2,81 & $(0,65-12,18)$ \\
\hline & Alto-medio & 0,60 & $(0,10-2,97)$ & $0,32^{\star}$ & $(0,14-0,72)$ & 1,23 & $(0,32-4,67)$ & 1,22 & $(0,32-4,69)$ \\
\hline \multirow{3}{*}{$\begin{array}{l}\text { Tercil de edad al comenzar el uso } \\
\text { de alcohol }{ }^{\mathrm{b}, \mathrm{c}} \text { (Ref: Tardío) }\end{array}$} & Temprano & - & & $0,34^{\star}$ & $(0,22-0,51)$ & 2,00 & $(0,61-6,87)$ & 1,63 & $(0,45-5,83)$ \\
\hline & Medio & - & & $0,49^{*}$ & $(0,38-0,64)$ & 1,24 & $(0,41-3,72)$ & 1,10 & $(0,36-3,32)$ \\
\hline & $X_{2}{ }_{2}[p]$ & - & & $37,78^{\star \star}$ & {$[<0,001]$} & 1,71 & {$[0,425]$} & 1,00 & {$[0,609]$} \\
\hline Tamaño de la muestra & Total $n^{d}$ & 1459 & & 1272 & & 1272 & & 1055 & \\
\hline
\end{tabular}

Nota. OR - odds-ratios; IC = Intervalo de confianza. Todos los análisis de regresión logísticas de tiempo discreto se basan en los datos ponderados de la Parte II persona-año controlando los grupos de edad persona-año de $<=15,16-17,18-20,21-24,25-29$ y 30+ (Ref). */** Significativo en el nivel 0,05, contraste bilateral. a Porcentaje de personas (/10) en la cohorte sexo-específica de +-5 años del individuo que había usado alcohol en el año previo para cada unidad persona-año. Ej. Para una mujer nacida en 1975, la cohorte correspondiente sería la de mujeres nacidas entre 1970 y 1980 , y un OR de 1.5 para inicio de uso se interpretaría como: para un $10 \%$ de incremento de personas en la cohorte edad-sexo que comenzó el uso en el año previo a la unidad persona año (controlando por todas las demás variables en el modelo), la probabilidad de comenzar el uso aumenta en un $50 \%$. ${ }^{b}$ El tercil de edad al comenzar el uso de alcohol se incluyó en todos los modelos excepto la transición al uso inicial. ' La edad de un individuo al comenzar el uso de alcohol se divide en terciles encuesta-específicas entre todos los que alguna vez hayan utilizado el alcohol. EL tercil más temprano (primera) es edad $s=15$, el 20 tercil es edad $16-17$ y el 3er tercil es edad $18+$ años. ${ }^{d} n=E l$ número total no ponderado de los encuestados incluidos en modelo condicionando la etapa inicial. e Los encuestados fueron excluidos del modelo de esta transición si el inicio de uso regular ocurrió después del inicio de abuso $(n=4)$. Los encuestados fueron excluidos del modelo de la transición si el comienzo de la etapa inicial ocurrió después del inicio de la segunda etapa. 
transición de uso a abuso. Un inicio más tardío en el uso de alcohol, comparado con otros encuestados, se asoció con mayores probabilidades de transición de uso a uso regular. La educación también se asociaba significativamente con las transiciones de uso y uso regular a abuso en estudiantes o personas con un nivel educativo bajo-medio, con mayores probabilidades de transición a trastorno en comparación con los individuos con un bajo nivel educativo.

\section{Transición a remisión desde abuso de alcohol}

La Tabla 3 presenta el análisis de supervivencia múltiple de tiempo discreto que examina las covariables de remisión desde abuso de alcohol incluyendo sexo, educación, tercil de edad de primer inicio, uso de alcohol de la cohorte nacimiento-sexo, número de años con el trastorno, y tercil de la velocidad de la transición desde uso a trastorno. La remisión de abuso de alcohol se asociaba significativamente con el nivel de uso de alcohol de la cohorte. Ser mujer y tener un nivel educativo bajo-medio a alto-medio se asociaba con un aumento de la probabilidad de remisión de abuso de alcohol. El tiempo con el trastorno, la velocidad de transición desde uso a abuso, y el tercil de inicio del uso de alcohol no se asociaban significativamente con la remisión.

\section{Discusión}

El presente estudio ha tratado de examinar la edad de inicio y el tiempo de transición entre varios estadios de uso de alcohol en un muestra representativa de la población general de la Región de Murcia, en el sureste de España, y a estimar la asociación de la cohorte de uso con la probabilidad de transición a uso de alcohol, trastorno, y remisión. Se obtuvieron varios resultados notables. Primero, los resultados indican que las tres cuartas partes de la población ha utilizado alcohol regularmente ( $\geq 12$ bebidas al año), una conducta establecida mayormente a los 20 años. En

Tabla 3. Asociaciones ajustadas de las variables socio-demográficas con transiciones del abuso de alcohol a remisión en Murcia.

\begin{tabular}{|c|c|c|c|}
\hline \multirow[t]{2}{*}{ Variable } & \multirow[t]{2}{*}{ Categoría } & \multicolumn{2}{|c|}{ Abuso (sin dependencia) a remisión de abuso ${ }^{\mathrm{e}}$} \\
\hline & & OR & IC $95 \%$ \\
\hline \multirow{2}{*}{ Sexo (Ref: Mujer) } & Varón & $0,23^{*}$ & $(0,13-0,38)$ \\
\hline & $X_{1}^{2}[p]$ & $31,22^{\star \star}$ & {$[<0,001]$} \\
\hline \multirow{2}{*}{ Porcentaje de uso de alcohol de la cohorte nacimiento-sexo ${ }^{a}$} & Uso de la cohorte & $2,02^{\star}$ & $(1,00-4,07)$ \\
\hline & $X_{1}^{2}[p]$ & $3,88^{\star \star}$ & {$[0,049]$} \\
\hline \multirow{5}{*}{ Nivel educativo (Ref: Alto) } & Estudiante & 1,49 & $(0,26-8,42)$ \\
\hline & Bajo & 3,14 & $(0,91-10,80)$ \\
\hline & Bajo-medio & $5,42^{\star}$ & $(2,33-12,59)$ \\
\hline & Alto-medio & $2,65^{\star}$ & $(1,22-5,77)$ \\
\hline & $X_{4}^{2}[p]$ & $92,25^{\star *}$ & {$[<0,001]$} \\
\hline \multirow[t]{2}{*}{ Tercil de edad al comenzar el uso de alcoholb (ref: Tardío) } & Medio & 1,10 & $(0,28-4,64)$ \\
\hline & $X_{2}{ }_{2}[p]$ & 3,31 & {$[0,192]$} \\
\hline \multirow{3}{*}{ Velocidad de transición de uso a trastornoc (Ref: Tardío) } & Temprano & 0,93 & $(0,40-2,21)$ \\
\hline & Medio & 1,12 & $(0,52-2,42)$ \\
\hline & $X_{2}^{2}[p]$ & 0,14 & {$[0,931]$} \\
\hline \multirow{2}{*}{ Tiempo con trastorno } & Años & 0,99 & $(0,95-1,03)$ \\
\hline & $X_{2}{ }_{2}[p]$ & 0,37 & {$[0,545]$} \\
\hline Tamaño de la muestra & Total $\left(N^{d}\right)$ & 63 & \\
\hline
\end{tabular}

Nota. OR - odds-ratios; IC = Intervalo de confianza. Todos los análisis de regresión logísticas de tiempo discreto se basan en los datos ponderados de la Parte II persona-año controlando los grupos de edad persona-año de $<=15,16-17,18-19,21-22,23-29$ y $30+$ (Ref). * ${ }^{* *}$ Significativo en el nivel o,05, contraste bilateral. a Porcentaje $(/ 10)$ de personas en la cohorte sexo-específica de +-5 años del individuo que había usado alcohol en el año previo para cada unidad persona-año. Ej. Para una mujer nacida en 1975, la cohorte correspondiente sería la de mujeres nacidas entre 1970 y 1980, y un OR de 1.5 para inicio de uso se interpretaría como: para un $10 \%$ de incremento de personas en la cohorte edad-sexo que comenzó el uso en el año previo a la unidad persona año (controlando por todas las demás variables en el modelo), la probabilidad de comenzar el uso aumenta en un $50 \%$. ${ }^{\text {b }}$ La edad de un individuo al comenzar el uso de alcohol se divide en terciles encuesta-específicas entre todos los que alguna vez hayan utilizado el alcohol. EL tercil más temprano (primera) es edad $\left\langle=15\right.$, el $2^{0}$ tercil es edad $16-17$ y el 3 er tercil es edad $18+$ años. ${ }^{c}$ La velocidad de transición de un individuo de uso de alcohol a trastorno se divide en terciles de encuesta-específicos. Al predecir la remisión de abuso, los terciles se calcularon para transición de uso a abuso: la más rápida a 0-3 años, el tercil medio a 4-9 años y transiciones tardías eran $10+$ años. ${ }^{d} \mathrm{n}=$ El número total no ponderado de los encuestados incluidos en el modelo condicionando la etapa inicial. e La remisión se define como informar de más de 12 meses, o por lo menos dos cumpleaños, desde el último trastorno relacionado con el problema. En los casos donde el tiempo desde el último problema era menos de dos años y la edad exacta de la remisión no se pudo definir, la edad de inicio de remisión se estableció como datos perdidos ( $n=10)$. Estos encuestados fueron excluidos del modelo de transición a la remisión. 
segundo lugar, los resultados señalan el rol de la cohorte nacimiento-sexo de uso como un factor asociado a la transición de una etapa de uso de alcohol a la siguiente. Por último, la remisión del abuso de alcohol se asoció con ser mujer, cohorte de uso y nivel educativo.

Consistente con los datos existentes en relación con la omnipresente naturaleza del uso de alcohol en las civilizaciones occidentales (Degenhardt et al., 2008) y con los datos de la (Observatorio Español de la Droga y las Toxicomanías, 2017), el uso de alcohol a lo largo de la vida se observó en cerca de nueve de cada diez residentes. Al cumplir los 16 años, un tercio de la población había tomado su primera copa, aumentando considerablemente a más de dos tercios al cumplir los 18 años, la edad legal para beber en España. Se encontró una rápida transición desde el primer uso al uso regular, con la mitad de los usuarios convirtiéndose en usuarios regulares al cabo de un año de su primera copa. Las tres cuartas partes de la población ha utilizado alcohol regularmente, lo cual puede complicar los esfuerzos preventivos para retrasar la edad de primer uso a pesar de su importante rol en la transición a los TCA (e.g., DeWit et al., 2000; Kalaydjian et al., 2009; Silveira et al., 2011). De hecho, el alcohol está disponible en los hogares, y las leyes actuales sobre la bebida no se aplican sistemáticamente en los establecimientos del sur de Europa que venden bebidas alcohólicas. El acceso fácil al alcohol podría asociarse con el hallazgo de que un tercio de los casos de abuso de alcohol y más uno de cada diez casos de dependencia ya había ocurrido al cumplir los 19 años. Más allá de la disponibilidad de alcohol para los jóvenes, estos resultados destacan la necesidad de realizar esfuerzos de prevención dirigidos a esta población, ya que se ha observado que los jóvenes con trastornos mentales tienen mayor riesgo de pasar a niveles superiores de uso de alcohol (Conway, Swendsen, Husky, He y Merikangas, 2016).

A pesar de lo habitual del uso regular de alcohol, definido como 12 o más copas al año, la prevalencia de por vida del abuso de alcohol sin dependencia $(4,9 \%)$ y de la dependencia del alcohol $(1,5 \%)$ es relativamente baja y dentro del rango de lo que se encuentra en otros países occidentales europeos (Alonso et al., 2004). En estos estudios, los índices de prevalencia dependen en gran medida de la prevalencia de los TCA en los varones $(8,6 \%$ y $2,5 \%$ para abuso de alcohol y dependencia, respectivamente) en comparación con las mujeres $(1,1 \%$ y $0,1 \%$ ) (Navarro-Mateu et al., 2015). En el presente estudio, ser varón estaba fuertemente asociado a un mayor riesgo de transición de uso a abuso y de uso regular a abuso, lo cual es consistente con los resultados de otras regiones del mundo (Cheng, Chandra, Alcover y Anthony, 2016; DeWit et al., 2000; Grant, 1997; Kalaydjian et al., 2009; Lee et al., 2009; Lopez-Quintero et al., 2011; Silveira et al., 2011; Suliman et al., 2010). Sin embargo, el sexo no se asociaba significativamente con el riesgo de transición de uso a uso regular ni tampoco con el inicio de uso de al- cohol, que en parte puede reflejar un acortamiento de las diferencias entre hombres y mujeres en el uso de sustancias observado en los últimos años (Slade et al., 2016).

La reciente evidencia con respecto a la importancia del consumo entre compañeros (Degenhardt et al., 2016) se confirmó en nuestro estudio, donde la cohorte nacimiento-sexo de uso de alcohol se asociaba significativamente con el inicio de uso y con la transición de uso de alcohol a uso regular y de uso regular a abuso. Este hallazgo sugiere que las medidas preventivas dirigidas a cohortes pueden resultar útiles en la reducción de la progresión a estadios posteriores de uso de alcohol. La cohorte nacimiento-sexo de uso de alcohol también se asociaba significativamente con la remisión de abuso, y subrayamos la importancia de normas sociales compartidas relacionadas con el uso de sustancias (Pollard, Freeman, Ziegler, Hersman y Goss, 2000). Combinado con los resultados relacionados con la velocidad a la que un individuo pasa a los estadios tempranos de uso de alcohol, los efectos de la cohorte de uso sugieren que los esfuerzos preventivos deberían orientarse hacia los jóvenes. Estos esfuerzos pueden centrarse en las escuelas o en las universidades, pero no sólo a estas localizaciones, ya que el uso de sustancias y los problemas de salud mental son por lo menos tan prevalentes, si no más, entre los jóvenes que ya no están en la escuela (Blanco et al., 2008; Degenhardt et al., 2016; Kovess-Masfety et al., 2016). Por último, mientras el consumo total global de alcohol per cápita se ha reducido a la mitad en las últimas décadas, pasando de cerca de 20 litros por año en 1975 a 11,2 litros en el 2010 (OMS, 2014), los cambios en los patrones de consumo, con una drástica reducción en el consumo de vino y un incremento en el consumo de la cerveza, pueden señalar vías adicionales para la prevención en España (Mateos, Páramo, Carrera y Rodríguez-López, 2002; Paschall, Grube y Kypri, 2009; Robledo de Dios, 2002).

En contraste con una cantidad significativa de pruebas en la literatura que muestran la importancia de la edad temprana del primer uso en el riesgo de transición a abuso de alcohol (Abdin et al., 2013; DeWit et al., 2000; Grant, Stinson y Harford, 2001; Kalaydjian et al., 2009), la edad temprana de inicio no se asociaba con la transición de uso o de uso regular a abuso ni tampoco con la remisión de abuso. Es más, se asociaba inversamente con el riesgo de transición de uso a uso regular. Como se mencionó anteriormente, determinados factores de riesgo tienen un efecto diferencial en el espectro total de los estadios de uso de alcohol (Kalaydjian et al., 2009; Lee et al., 2009; Silveira et al., 2011; Suliman et al., 2010). Estudios previos sugieren de hecho que la edad temprana de uso de alcohol se asociaba con algunos, pero no con todos, los estadios de uso de alcohol (Silveira et al., 2011), y que puede no afectar la rápida transición a trastornos de alcohol entre adolescentes o jóvenes varones (Cheng et al., 2016). Se requieren investigaciones adicionales para documentar el rol diferencial de 
los factores clave asociados a mayor riesgo de transición a los TCA en España, incluyendo el rol de la edad temprana de inicio en el uso de alcohol.

Caben señalar varias limitaciones al interpretar los resultados. Primero, había tasas insuficientes de dependencia del alcohol en la muestra $(1,5 \%)$ para examinar las asociaciones de transición de uso a dependencia, o de dependencia a remisión. Los datos disponibles para el modelo de transición a remisión de abuso a través de los resultados se basan en un pequeño número de casos de abuso (N=63) y se deben interpretar los resultados con precaución. Dicho esto, las tasas de uso y abuso de alcohol fueron suficientes para examinar los inicios respectivos. Segundo, la edad de inicio para los estadios de uso de alcohol se basaba en auto-informes retrospectivos y que pueden haber estado influidos por un sesgo de memoria (Johnson y Schultz, 2005; Shillington, Woodruff, Clapp, Reed y Lemus, 2012). Sin embargo, se ha demostrado que el diseño estructurado del instrumento diagnóstico combinado con la estrategia utilizado en las encuestas WMH han reducido este sesgo de memoria (Knäuper, Cannell, Schwarz, Bruce y Kessler, 1999). Por último, como el estudio se llevó a cabo en una muestra representativa de una sola región de España, se requieren investigaciones adicionales para replicar los resultados en muestras de otras zonas geográficas.

El presente estudio ha documentado la edad de inicio del espectro completo de estadios de uso de alcohol desde el primer uso a la dependencia y remisión en la población general de la Región de Murcia. Hasta donde llega nuestro conocimiento, los resultados son los primeros en medir el tiempo de la evolución natural del uso de alcohol en la población general de una región de Europa meridional. Los resultados resaltan los períodos sensibles con transiciones rápidas a niveles de uso de alcohol más altos. El estudio enfatiza además la importancia del uso de cohortes en todo el espectro de estadios de uso de alcohol, lo que aporta datos importantes para los responsables de la prevención de los problemas relacionados con el alcohol en España.

\section{Agradecimientos}

Los autores dan las gracias a todos participantes por su colaboración, al Observatorio sobre Drogas de la Región de Murcia y reconocen el apoyo de Carlos Giribert Muñoz en el desarrollo del proyecto PEGASUS-Murcia cuando él era el Director Adjunto de Programas, Cronicidad y Innovación del Salud Autoridad de Murcia.

\section{Financiación}

El proyecto PEGASUS-Murcia fue apoyado por las Autoridades Regionales de Salud de Murcia ("Servicio Murciano de Salud and Consejería de Sanidad y Política Social”) (Decreto $n^{\circ} 455 / 2009$ ) y la "Fundación para la Formación e Investigación Sanitarias (FFIS) de la Región de Murcia" ( ${ }^{\circ}$ Expedientes: CM0829 I and FFIDS/EMER09/14). El proyecto PEGASUS-Murcia se realiza en conjunto con el WHOWMH Survey Initiative. El trabajo de C. Bharat fue apoyado por una beca del Australian National Health and Medical Research Council (NHMRC) (no. 1081984). Los autores dan las gracias al personal del WMH Coordinating Center en las Universidades de Harvard y Michigan por su asistencia con la instrumentación, trabajo de campo y análisis de datos. Estas actividades fueron apoyadas por el United States National Institute of Mental Health (R01MH070884), la Fundación John D. and Catherine T. MacArthur, la Fundación Pfizer, el U.S. Public Health Service (R13-MH066849, R01-MH069864, and R01 DA016558), el Fogarty International Center (FIRCA R03- TW006481), la Pan American Health Organization, la Fundación Eli Lilly y Company, Ortho-McNeil Pharmaceutical, Inc., GlaxoSmithKline, Bristol-Myers Squibb and Shire. La lista completa de publicaciones de WMH puede encontrarse en http://www.hcp.med.harvard.edu/wmh/. Los fundadores directos e indirectos no desempeñaron ningún rol en el diseño del estudio, la recogida y el análisis de la información, la decisión de publicar, o la preparación del manuscrito.

\section{Conflicto de intereses}

E1 PEGASUS-Murcia proyecto se realiza en conjunto con la WHO-World Mental Health (WMH) Survey Initiative. El personal del WMH Coordinating Center en las universidades de Michigan y Harvard ayudó con la instrumentación, el trabajo de campo y el análisis de datos. Estas actividades fueron apoyados parcialmente por Ortho-McNeil Pharmaceutical, Inc., GlaxoSmithKline, Bristol-Myers Squibb and Shire. En los últimos tres años, Dr. Kessler ha sido consultor para Hoffman-La Roche, Inc., Johnson y Johnson Wellness and Prevention, and Sonofi-Aventis Groupe. Dr. Kessle ha sido miembro de los consejos asesores de Mensante Corporation, Plus One Health Management, Lake Nona Institute, and U.S. Preventive Medicine. Dr. Kessler es copropietario de DataStat, Inc. No hay patentes, productos de desarrollo o productos comercializados que declarar.

\section{Referencias}

Abdin, E., Subramaniam, M., Vaingankar, J. A. y Chong, S. A. (2013). The role of sociodemographic factors in the risk of transition from alcohol use to disorders and remission in singapore. Alcohol and Alcoholism, 49, 103-108. doi:10.1093/alcalc/agt126.

Alonso, J., Angermeyer, M. C., Bernert, S., Bruffaerts, R., Brugha, T. S., Bryson, H., ... Vollebergh, W. (2004). Prevalence of mental disorders in europe: Results from the European Study of the Epidemiology of Mental Disor- 
ders (ESEMeD) project. Acta Psychiatrica Scandinavica, 109, 21-27. doi:10.1111/j.1600-0047.2004.00327.x.

American Psychiatric Association. (2000). Diagnostic and statistical manual of mental disorders (Fourth edition - Text revision). Washington, DC: American Psychiatric Association.

Blanco, C., Okuda, M., Wright, C., Hasin, D. S., Grant, B. F., Liu, S.-M. y Olfson, M. (2008). Mental health of college students and their non-college-attending peers: Results from the national epidemiologic study on alcohol and related conditions. Archives of General Psychiatry, 65, 1429-1437. doi:10.1001/archpsyc.65.12.1429.

Carballo, J. L., Fernandez-Hermida, J. R., Secades-Villa, R. y García-Rodríguez, O. (2008). Determinants of recovery from alcohol problems in treated and untreated individuals in a spanish sample. Adicciones, 20, 49-58.

Carballo, J. L., Fernández-Hermida, J. R., Sobell, L. C., Dum, M., Secades-Villa, R., García-Rodríguez, O., ... AlHalabí-Diaz, S. (2008). Differences among substance abusers in spain who recovered with treatment or on their own. Addictive Behaviors, 33, 94-105. doi:10.1016/j. addbeh.2007.07.013.

Cheng, H. G., Chandra, M., Alcover, K. C. y Anthony, J. C. (2016). Rapid transition from drinking to alcohol dependence among adolescent and young-adult newly incident drinkers in the united states, 2002-2013. Drug $\mathcal{E}$ Alcohol Dependence, 168, 61-68. doi:10.1016/j.drugalcdep.2016.08.015.

Conway, K. P., Swendsen, J., Husky, M. M., He, J.P. y Merikangas, K. R. (2016). Association of lifetime mental disorders and subsequent alcohol and illicit drug use: Results from the national comorbidity survey-adolescent supplement. Journal of the American Academy of Child E Adolescent Psychiatry, 55, 280-288. doi:10.1016/j.jaac.2016.01.006.

Degenhardt, L., Chiu, W.-T., Sampson, N., Kessler, R. C., Anthony, J. C., Angermeyer, M., ... Huang, Y. (2008). Toward a global view of alcohol, tobacco, cannabis, and cocaine use: Findings from the WHO World Mental Health surveys. PLoS Medicine, 5, e141. doi:10.1371/ journal.pmed.0050141.

Degenhardt, L., Stockings, E., Patton, G., Hall, W. D. y Lynskey, M. (2016). The increasing global health priority of substance use in young people. Lancet Psychiatry, 3, 251-264. doi:10.1016/S2215-0366(15)00508-8.

Demyttenaere, K., Bruffaerts, R., Posada-Villa, J., Gasquet, I., Kovess, V., Lepine, J. P., ... WHO World Mental Health Survey Consortium. (2004). Prevalence, severity, and unmet need for treatment of mental disorders in the World Health Organization World Mental Health surveys. Journal of the American Medical Association, 291, 25812590. doi:10.1001/jama.291.21.2581.

DeWit, D. J., Adlaf, E. M., Offord, D. R. y Ogborne, A. C. (2000). Age at first alcohol use: A risk factor for the development of alcohol disorders. American Journal of Psychiatry, 157, 745-750. doi:10.1176/appi.ajp.157.5.745.
Flórez-Salamanca, L., Secades-Villa, R., Hasin, D. S., Cottler, L., Wang, S., Grant, B. F. y Blanco, C. (2013). Probability and predictors of transition from abuse to dependence on alcohol, cannabis, and cocaine: Results from the national epidemiologic survey on alcohol and related conditions. The American Journal of Drug and Alcohol Abuse, 39, 168-179. doi:10.3109/00952990.2013.772618.

Grant, B. F. (1997). Prevalence and correlates of alcohol use and DSM-IV alcohol dependence in the United States: Results of the national longitudinal alcohol epidemiologic survey. Journal of Studies on Alcohol, 58, 464-473. doi:10.15288/jsa.1997.58.464.

Grant, B. F., Stinson, F. S. y Harford, T. C. (2001). Age at onset of alcohol use and DSM-IV alcohol abuse and dependence: A 12-year follow-up. Journal of Substance Abuse, 13, 493-504. doi:10.1016/S0899-3289(01)00096-7.

Gual, A., Arbesú, J. Á., Zarco, J., López-Pelayo, H., Miquel, L. y Bobes, J. (2016). Alcoholism and its treatment approach from a citizen perspective. Adicciones, 28. 163173. doi:10.20882/adicciones.742.

Johnson, E. O. y Schultz, L. (2005). Forward telescoping bias in reported age of onset: An example from cigarette smoking. International Journal of Methods in Psychiatric Research, 14, 119-129. doi:10.1002/mpr.2.

Kalaydjian, A., Swendsen, J., Chiu, W.-T., Dierker, L., Degenhardt, L., Glantz, M., ... Kessler, R.C. (2009). Sociodemographic predictors of transitions across stages of alcohol use, disorders and remission in the national comorbidity survey-replication. Comprehensive Psychiatry, 50, 299-306. doi:10.1016/j.comppsych.2008.09.012.

Kessler, R.C. y Ustun, T. B. (2004). The World Mental Health $(\mathrm{WMH})$ survey initiative version of the World Health Organization (WHO) Compositve International Diagnostic Interview (CIDI). International Journal of Methods in Psychiatric Research, 13, 93-121. doi:10.1002/mpr.168.

Knäuper, B., Cannell, C. F., Schwarz, N., Bruce, M. L. y Kessler, R. C. (1999). Improving accuracy of major depression age-of-onset reports in the U.S. National Comorbidity Survey. International Journal of Methods in Psychiatric Research, 8, 39-48. doi:10.1002/mpr.55.

Kovess-Masfety, V., Leray, E., Denis, L., Husky, M., Pitrou, I. y Bodeau-Livinec, F. (2016). Mental health of college students and their non-college-attending peers: Results from a large french cross-sectional survey. BMC Psychology, 4. doi:10.1186/s40359-016-0124-5.

Lee, S., Guo, W. J., Tsang, A., He, Y. L., Huang, Y. Q., Zhang, M. Y., ... Kessler, R. C. (2009). Associations of cohort and socio-demographic correlates with transitions from alcohol use to disorders and remission in metropolitan China. Addiction, 104, 1313-1323. doi:10.1111/j.13600443.2009.02595.x.

Lev-Ran, S., Imtiaz, S., Rehm, J. y Le Foll, B. (2013). Exploring the association between lifetime prevalence of mental illness and transition from substance use to 
substance use disorders: Results from the National Epidemiologic Survey of Alcohol and Related Conditions (NESARC). The American Journal on Addictions, 22, 93-98. doi:10.1111/j.1521-0391.2013.00304.x.

Lopez-Quintero, C., Cobos, J. P., Hasin, D. S., Okuda, M., Wang, S., Grant, B. F. y Blanco, C. (2011). Probability and predictors of transition from first use to dependence on nicotine, alcohol, cannabis, and cocaine: Results of the National Epidemiologic Survey on Alcohol and Related Conditions (NESARC). Drug and Alcohol Dependence, 115, 120-130. doi:10.1016/j.drugalcdep.2010.11.004.

Mateos, R., Páramo, M., Carrera, I. y Rodríguez-López, A. (2002). Alcohol consumption in a southern european region (Galicia, Spain). Substance Use E Misuse, 37, 1957-1976. doi:10.1081/JA-120016226.

Navarro-Mateu, F., Tormo, M., Vilagut, G., Alonso, J., Ruiz-Merino, G., Escamez, T., ... Navarro, C. (2013a). Epidemiology and genetics of common mental disorders in the general population: The PEGASUS-Murcia project. BMJ Open, 3. doi:10.1136/bmjopen-2013-004035.

Navarro-Mateu, F., Morán-Sánchez, I., Alonso, J., Tormo, M. J., Pujalte, M. L., Garriga, A., ... Navarro, C. (2013b). Cultural adaptation of the latin american version of the World Health Organization Composite International Diagnostic Interview (CIDI v 3.0) for use in Spain. Gaceta Sanitaria, 27, 325-331. doi:10.1016/j.gaceta.2012.06.005.

Navarro-Mateu, F., Tormo, M. J., Salmerón, D., Vilagut, G., Navarro, C., Ruíz-Merino, G., ... Kessler, R. C. (2015). Prevalence of mental disorders in the south-east of spain, one of the european regions most affected by the economic crisis: The cross-sectional pegasus-murcia project. PLoS One, 10. doi:10.1371/journal.pone.0137293.

Oberleitner, L. M., Smith, P. H., Weinberger, A. H., Mazure, C. M. y McKee, S. A. (2015). Impact of exposure to childhood maltreatment on transitions to alcohol dependence in women and men. Child Maltreatment, 20, 301-308. doi:10.1177/1077559515591270.

Observatorio Español de la Droga y las Toxicomanías. (2017). Edades 2015-2016. Encuesta sobre alcohol y drogas en españa. Madrid: Delegación del gobierno para el plan nacional sobre drogas. Recuperado de http://www. pnsd.msssi.gob.es/profesionales/sistemasInformacion/ sistemaInformacion/pdf/2015_EDADES_Informe_.pdf.

Paschall, M. J., Grube, J. W. y Kypri, K. (2009). Alcohol control policies and alcohol consumption by youth: A multi-national study. Addiction, 104, 1849-1855. doi:10.1111/ j.1360-0443.2009.02698.x.

Pérez, B. (2002). El alcohol como problema de salud pública. La responsabilidad de los poderes públicos. Adicciones, 14, 291-301.

Pollard, J. W., Freeman, J. E., Ziegler, D. A., Hersman, M. N. y Goss, C. W. (2000). Predictions of normative drug use by college students. Journal of College Student Psychotherapy, 14, 5-12. doi:10.1300/J035v14n03_03.
Probst, C., Moyo, D., Purshouse, R. y Rehm, J. (2015). Transition probabilities for four states of alcohol use in adolescence and young adulthood: What factors matter when? Addiction, 110, 1272-1280. doi:10.1111/ add.12985.

Rehm, J., Anderson, P., Barry, J., Dimitrov, P., Elekes, Z., Feijão, F., ... Kraus, L. (2015). Prevalence of and potential influencing factors for alcohol dependence in Europe. European Addiction Research, 21, 6-18. doi:10.1159/000365284.

Rehm, J., Gmel, G. y Gual, A. (2013). Alcohol consumption, alcohol dependence and related harms in Spain, and the effect of treatment-based interventions on alcohol dependence. Adicciones, 25, 11-18.

Rehm, J., Mathers, C., Popova, S., Thavorncharoensap, M., Teerawattananon, Y. y Patra, J. (2009). Global burden of disease and injury and economic cost attributable to alcohol use and alcohol-use disorders. The Lancet, 373, 2223-2233. doi:10.1016/S0140-6736(09)60746-7.

Robledo de Dios, T. (2002). Políticas institucionales de prevención de los problemas de salud generados por el consumo de bebidas alcohólicas en España y Europa. Adicciones, 14, S303-S315.

Shillington, A. M., Woodruff, S. I., Clapp, J. D., Reed, M. B. y Lemus, H. (2012). Self-reported age of onset and telescoping for cigarettes, alcohol, and marijuana: Across eight years of the national longitudinal survey of youth. Journal of Child Eं Adolescent Substance Abuse, 21, 333-348. doi:10.1080/1067828X.2012.710026.

Silveira, C. M., Viana, M. C., Siu, E. R., de Andrade, A. G., Anthony, J. C. y Andrade, L. H. (2011). Sociodemographic correlates of transitions from alcohol use to disorders and remission in the Sao Paulo megacity mental health survey, brazil. Alcohol and Alcoholism, 46, 324-332. doi:10.1093/alcalc/agr007.

Slade, T., Chapman, C., Swift, W., Keyes, K., Tonks, Z. y Teesson, M. (2016). Birth cohort trends in the global epidemiology of alcohol use and alcohol-related harms in men and women: Systematic review and metaregression. BMJ Open, 6. doi:10.1136/bmjopen-2016-011827.

Suliman, S., Seedat, S., Williams, D. R. y Stein, D. J. (2010). Predictors of transitions across stages of alcohol use and alcohol-use disorders in South Africa. Journal of Studies on Alcohol and Drugs, 71, 695-703. doi:10.15288/ jsad.2010.71.695.

von Elm, E., Altman, D.G., Egger, M., Pocock, S.J., Gotzsche, P.C. y Vandenbroucke, J.P. (2007). The Strengthening the Reporting of Observational Studies in Epidemiology (STROBE) statement: guidelines for reporting observational studies. PLoS Med, 4: e296. doi:10.1371/ journal.pmed.0040296.

Werner, K. B., Sartor, C. E., McCutcheon, V. V., Grant, J. D., Nelson, E. C., Heath, A. C. y Bucholz, K. K. (2016). Association of specific traumatic experiences with alco- 
hol initiation and transitions to problem use in european american and african american women. Alcoholism: Clinical and Experimental Research, 40, 2401-2408. doi:10.1111/acer.13220.

World Health Organization. (2014). Global status report on alcohol and health. Geneva, Switzerland: World Health Organization. 